\title{
«Excellence, un libéral peut avoir des vertus ! ": stabilité, instabilité et plasticité de la forme sémantique libéral(isme)
}

\author{
Julien Longhi \\ Université François Rabelais de Tours \\ Laboratoire de Recherche sur le Langage (Clermont-Fd II) \\ longhi@1rl.univ-bpclermont.fr
}

\section{Introduction}

Dans les discours politiques contemporains, l'emploi des lexèmes libéral et libéralisme (et de leurs dérivés) fait l'objet d'enjeux argumentatifs, et conduit à des luttes symbolico-idéologiques parfois violentes. Dans ce contexte, les sens de libéral, apparus en 1160 (qui ne rencontre pas ou qui n'impose pas de contraintes, de limites; qui n'impose pas ou qui n'accepte pas que soit imposées à autrui certaines contraintes; ou favorable au libéralisme économique) semblent parfois bien désuets. Dans une conception dynamique de la construction du sens en discours, inspirée de la Théorie des Formes Sémantiques (TFS, de Cadiot et Visetti), nous souhaitons analyser les mécanismes de constitution du sens de libéral(isme), afin de relever, en diachronie comme en synchronie, la stabilité et l'instabilité repérables dans les usages. Pour cela, nous étudions deux corpus distincts : un corpus constitué des textes de Balzac et de Stendhal, et un corpus composé de discours prononcés par Le Pen, Chirac et Madelin de 1997 à 2004.

\section{Une approche dynamique de la construction du sens en discours}

L'objet que nous envisageons, libéral(isme), sera appréhendé dans cet article selon le double régime d'indexicalité et d'argumentativité du sens.

\subsection{La TFS et l'indexicalité du sens}

Nous adoptons une conception du langage qui se situe dans la lignée de la phénoménologie de la perception, développé en particulier en France par Merleau-Ponty :

\begin{abstract}
Chaque acte linguistique partiel comme partie d'un tout et acte commun du tout de la langue, ne se borne pas à en dépenser le pouvoir, il le recrée parce qu'il nous fait vérifier, dans l'évidence du sens donné et reçu, la capacité qu'ont les sujets parlants de dépasser les signes vers le sens, dont après tout ce que nous appelons la langue n'est que le résultat visible et l'enregistrement. Les signes n'évoquent pas seulement pour nous d'autres signes, et cela sans fin, le langage n'est pas comme une prison où nous soyons enfermés ou un guide dont nous aurions à suivre aveuglément les indications, parce que leur usage actuel, à l'intersection de ces mille gestes apparaît enfin ce qu'ils veulent dire, et à quoi ils nous ménagent un accès si facile que nous n'aurons plus même besoin d'eux pour nous y référer $(1969,146)$.
\end{abstract}

Dans cette perspective, le sens n'existe pas en langue mais est constitué par l'activité langagière, et entre en relation avec notre perception et notre expérience ${ }^{1}$. Dans la TFS, les dynamiques sémantiques peuvent êtres saisies selon trois phases ou régimes de sens, appelés motifs, profils et thèmes. Cadiot et Visetti définissent « les motifs linguistiques comme des germes de signification chaotiques et/ou instables, et le profilage comme un système, déjà frayé et enregistré en lexique et en grammaire, de parcours de stabilisations différentielles pour des lexèmes en interdéfinition » (2001-2, 6). La thématique, à prendre dans un sens textuel, renvoie à «ce dont on parle », à l'ensemble de ce qui est «posé » par l'activité de 
langage. Les motifs prennent en compte une certaine couche morphémique du sens (tout en se concevant également au niveau du mot); les opérations de profilages stabilisent les unités, et plongent les objets dans la voie des thématiques; et les thèmes se disposent au niveau textuel.

Le développement théorique de la TFS doit, dans notre perspective discursive, pouvoir intégrer les mécanismes de mise en discours des unités. C'est pourquoi notre démarche s'intéressera à certains aspects de la pragmatique intégrée (Ducrot) : nous justifierons alors la substitution du concept de thème par celui de topos.

\subsection{Une dimension argumentative et énonciative}

La Théorie de l'argumentation dans la langue, et la Théorie des topoï qui lui a succédée, entendaient montrer dans quelle mesure la pragmatique intervient sur la sémantique. Pour Ducrot et Anscombre, il n'y a pas de sens littéral en tant que ce sens littéral serait une constante sémantique. Le noyau sémantique profond des énoncés est constitué par des relations qu'entretient cet énoncé avec les discours qui le précèdent et le suivent. Ces relations sont argumentatives, et la relation d'argument à conclusion est de nature gradable. Lors d'une énonciation, le locuteur donne des indications sur le chemin qu'il a choisi pour aller d'un argument à une conclusion : ce sont les topoï, c'est-à-dire des principes généraux qui servent d'appui au raisonnement mais qui ne sont pas le raisonnement. Ils sont utilisés, pas assertés, et ils sont présentés comme faisant l'objet d'un consensus au sein d'une communauté. Ils peuvent être créés de toute pièce, issus d'une idéologie, d'une donnée sociologique. L'étude de leur nature permet de distinguer les topoï intrinsèques qui fondent la signification d'une unité lexicale - c'est-à-dire qu'ils sont potentiellement présents dans les unités lexicales - et les topoï extrinsèques qui sont utilisés pour fonder des enchaînements conclusifs. Ce concept de topos permet de rendre compte de manière argumentative des enchaînements en discours. Cependant, cette théorie présente une contradiction forte face au préambule dans lequel nous inscrivions cet article: la non-indexicalité du sens. En effet, dans une perspective phénoménologique, le mot, dans le discours, n'est pas mis d'abord en situation de décrire mais de créer des conditions dont on attend qu'elles contribuent à rendre possible un acte de référence : « la face descriptive du mot est subordonnée à cette fonction d'index de discours » (Cadiot 1997, 245). En outre, les corpus discursifs témoignent de la diversité du sens et des dynamiques sémantiques : il devient alors difficile de définir la nature des topoï de manière aussi nette que dans les théories argumentatives. Cette conception de la constitution du sens inverse le rapport traditionnellement attribué entre les objets du langage et leur référents :

\footnotetext{
La notion de référent peut alors être généralisée à celle d'《objet». L'«objet» est défini comme une infinité potentielle de rapports focalisés sur un point. Cette infinité potentielle est l'expression d'une conception d'« équilibre phénoménologique », qui établit qu'un objet est une synthèse d'apparences. Ceci constitue le renversement fondamental à la théorie : on ne peut pas dire qu'un objet prend des apparences, mais que des apparences - parce qu'elles sont conçues comme telles — synthétisent un objet. [...] L'articulation de ces notions jette les bases d'une théorie du sens que j'ai nommée « indexicalité du sens » (Lebas, 1999, 487-488).
}

Nous souhaitons donc développer une analyse sémantico-discursive, ayant pour soucis de relever les mécanismes discursifs qui rendent possible ces synthèses d'apparences. En effet, nous reconnaîtrons, à la suite de G.-E. Sarfati (1996), le primat méthodologique de l'analyse des ensembles discursifs, privilégiés puisqu'ils définissent aussi bien des lieux d'inscription que des modélisations spontanées du système du sens commun. Ces ensembles discursifs doivent permettre de décrire non seulement les parcours de constitution des unités linguistiques, mais également de rapporter ces parcours aux formations qui en sont à l'origine, afin de définir les contours d'une topique ambiante relative à un objet particulier. L'inscription de la doxa dans la langue est postulée relativement à l'organisation d'un système du sens commun lui-même régi par un dispositif de topoï. Le sens commun est le concept organisateur du paradigme topique, il désigne l'ensemble des normes investies par les sujets dans les pratiques sociodiscursives. Le concept de topos serait son concept général descriptif. On réservera le concept de doxa à la délimitation d'une région du sens commun, comme telle dotée de son dispositif de topoï spécifiques. 
L'ambition affichée ici est de caractériser différentes positions énonciatives par les topoï qu'elles partagent. Ceci permet de repenser la notion déjà ancienne de Formation Discursive, dans une perspective sémantique. Pour Foucault, ce qui est décrit sous le nom de formation discursive, ce sont, au sens strict, des groupes d'énoncés, c'est-à-dire des ensembles de performances verbales qui sont reliées au niveau des énoncés : dans notre travail, la caractérisation d'une formation discursive se ferait au plan sémantique. A ce titre, la caractérisation sémantico-discursive des unités linguistiques s'incarne dans le concept d'objet discursif (Longhi 2007 et 2008) : ces synthèses d'apparences qui définissent un objet ne sont pas seulement celles du monde d'expérience, elles comprennent aussi, comme nous l'avons dit, les mécanismes discursifs qui viennent à produire leurs propres normes, d'où l'appellation objet discursif. Cette approche, appliquée ici à libéral(isme), fonctionne aussi bien pour d'autres unités lexicales. Ainsi, Cadiot et Nemo ont publié un nombre conséquent d'articles appuyant cette démarche indexicale (sans le versant argumentatif) à partir de termes tels que mur, boîte, etc. De notre côté, nous avons développé cette méthodologie à propose de livre (Longhi à paraître), et cette démarche énonciative et indexicale ne se heurte pas à ce changement : nous ne différencions bien entendu pas les noms dits abstraits d'autres qui seraient considérés comme concrets; d'ailleurs, le fait qu'un objet possède ou non une contrepartie tangible ne modifie par le positionnement adopté face à la constitution du sens.

\section{Du latin au 19ème siècle : constitution du potentiel sémantique}

En croisant diverses sources lexicographiques (Le Robert, Le Robert Historique, le TLFI), nous savons que libéral est emprunté au latin liberalis, qui vient de liber.

\subsection{Etymologie, morphologie et perception morphologique}

Liber signifiait en latin «de condition libre, non occupé, sans entrave ». L'adjectif qui se forme sur ce nom est liberalis, dont la signification touche trois domaines distincts, qui interagissent pourtant : d'une manière générale il signifie "relatif à une personne de condition libre ", "affaire où la condition d'un homme libre est en jeu », ou «qui sied à une personne de condition libre »; par rapport au physique, il signifie «noble, gracieux, bienséant»; dans le domaine moral, il se rapproche de "noble, honorable, généreux, libéral sous le rapport de l'argent ». Ainsi, le changement de catégorie d'appartenance est très riche au niveau sémantique, puisque les sens de liberalis sont conditionnés par (tout en anticipant sur) les domaines et contextes d'application. Ce premier passage, en latin, de la catégorie nominale à la catégorie adjectivale permet d'enregistrer certains traits : liberalis conserve les sens relatifs à liber (dans son acception générale), mais peut également porter plus particulièrement sur le physique ou sur l'aspect moral. Ainsi c'est plutôt l'aspect «sans entrave » de liber qui sera mis en saillance pour l'application particulière au physique ou aux qualités morales de quelqu'un : l'absence d'entraves sera alors comprise dans le cadre de l'interaction avec autrui, le physique ou la moralité d'une personne ne venant pas « faire entrave » à l'appréciation que l'on en aurait.

Libéral ${ }^{2}$ pourrait être également perçu comme une suffixation en -al de liber : ce serait la suffixation d'une base latine. Qu'il soit directement formé sur liberalis n'évacue pas totalement le sémantisme de l'antécédent de liberalis, liber, combiné à -al. La perception morphologie (voir Longhi 2006 à ce sujet) agit de cette manière : par association à d'autres lexèmes comme liberté, libération, l'impression de construction propre à libéral est obtenue, par identification d'une base commune, liber-, qui est précisément le « morphème » d'origine : 
Étymologie:

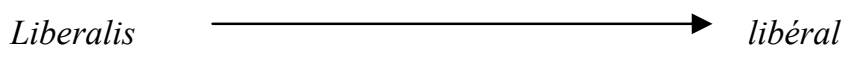

Position dans le lexique:

$$
\text { Liber }+ \text {-al libéral }
$$

Le suffixe - al peut simplement véhiculer « relatif à, qui se rapporte à, qui appartient à, qui concerne », et dans ce cas c'est le rapport que la morphologie souligne ; mais si -al sert à exprimer une qualité ou un défaut, l'argumentativité du terme est introduite dès la formation morphologique. $\mathrm{Ce}-a l$ servirait à exprimer une qualité (ou un défaut) : le « défaut d'être sans entrave », ou la «qualité d'être libre », etc. C'est ainsi que libéral, dans la langue française, sera considéré comme synonyme de généreux, ou se rencontrera dans professions libérales (exercées librement), ou pourra désigner quelqu'un qui est favorable aux libertés individuelles, dans le domaine politique, économique et social. Les différents domaines d'application de ce lexème sont aussi à prendre en compte, car ils peuvent entretenir des relations, et exercer une influence les uns sur les autres. On peut envisager que le sens " généreux » de liberalis dans le domaine moral s'intègre au motif général de libéral, et reste présent, peut-être en arrière plan, lors de l'actualisation des sens propres aux autres domaines, comme le politique ou socioéconomique.

\subsection{La politisation des sens au $19^{\text {ème }}$ siècle : illustration chez Balzac et Stendhal}

Il apparaît que le $19^{\text {ème }}$ siècle, dans le contexte post-révolutionnaire qui le caractérise, va créditer le lexème libéral d'acceptions originales, qui synthétisent les évolutions tant linguistiques qu'historiques, pour constituer une période de figement sémantique. Cette période est également ouverte aux innovations sémantiques qui seront connues jusqu'à nos jours. Pour rendre compte de cela, nous comparons les attestations de libéral chez Balzac et Stendhal ${ }^{3}$, dans le corpus de textes recueillis dans la banque de données Frantext. 78 occurrences sont recensées chez Balzac, et 61 chez Stendhal. Pour pouvoir accéder au motif sous-jacent à tous ces sens, nous relevons dans un premier temps les grandes thématiques dans lesquelles les occurrences de libéral s'intègrent. En effet, au niveau méthodologique, le nombre important d'occurrences fournies par Frantext, ainsi que la recension d'emplois plus ou moins figés, nous a incité à regrouper les énoncés selon les thématiques qu'ils mobilisent (un même énoncé pourrait appartenir à plusieurs thématiques, ce qui est une conséquence directe du dynamicisme langagier). Lors de cette procédure, le regroupement des énoncés s'est avéré bien plus simple pour le sous-corpus Balzac: les collocations y sont en effet plus récurrentes et plus apparentes, tandis que les énoncés provenant du souscorpus Stendhal posent parfois des problèmes d'interprétation. Le graphique suivant indique le pourcentage d'occurrences regroupées dans chaque thématique, pour chaque corpus: 


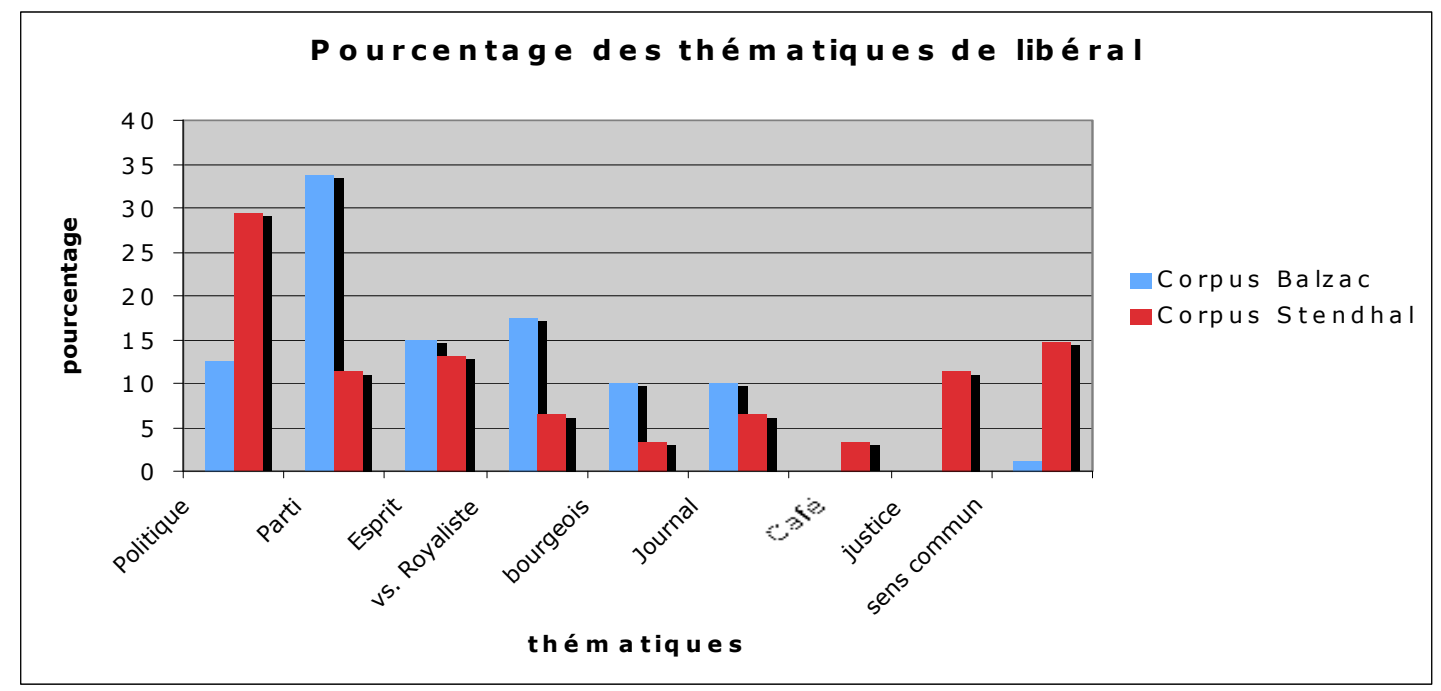

Graphique $\mathrm{n}^{\circ} 1: \quad$ pourcentage des thématiques de libéral

Le corpus Balzac se distingue par les emplois de libéral liés à parti, des emplois en opposition à royaliste (ou équivalent), et également - dans une moindre mesure - à la thématique /journal/. Chez Stendhal, les acceptions politiques sont plus nombreuses, tout comme les liens avec la thématique/justice/. En outre, les emplois de sens communs y sont beaucoup plus représentés. Pour expliciter plus précisément toutes ces différences, nous procéderons tout d'abord à l'étude de chaque sous-corpus.

Le schéma suivant synthétise les différentes thématiques qui sont mobilisées chez Balzac, en affinant tant que possible celles relevées sur le graphique général précédent :

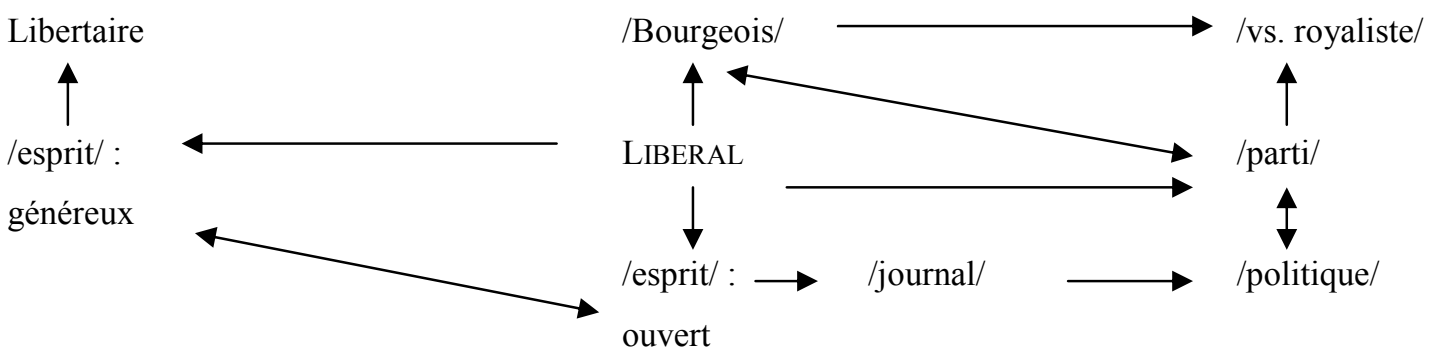

Schéma $\mathrm{n}^{\circ} 1: \quad$ Thématiques dans le corpus Balzac

Comme nous essayons de le montrer, ces thématiques sont étroitement liées entre elles, ce qui est d'une grande importance dans un cadre dynamique de la construction sémantique : les stabilisations sont en effet à voir au sein d'un continu sémantique, et non pas d'un système à l'intérieur duquel les unités seraient juxtaposées. Une fois le repérage de ces thématiques effectué, il reste à repérer les domaines d'application de l'objet, et les pôles qui tendent à figer une certaine identité aux objets. A partir du schéma précédent, il nous semble que libéral est construit dans trois grands domaines : [moral], [social] et [politique], étant entendu que les sens issus de ces différents domaines "communiquent», sont enregistrés par le motif, et sont plus ou moins saillants selon les contextes d'emploi. En mettant en perspective ces attestations avec la réflexion étymologique et historique effectuée, nous pensons que le motif qui sous-tend toutes les dynamiques sémantiques est //ouverture//. 
Dans le domaine [politique], nous relevons des profilages en parti libéral, des positionnements dans le champ (vie politique, journalisme), et des oppositions à royaliste. Par exemple :

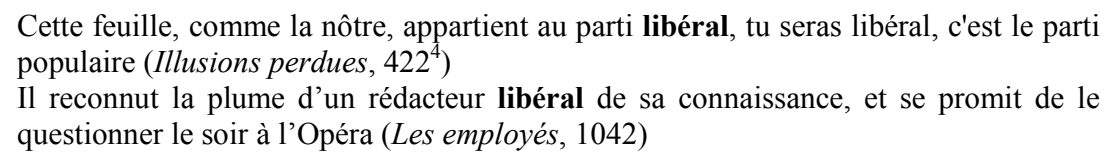

En plus de la quantité d'attestations plus importante dans la thématique/vs. royaliste/ chez Balzac, ce sous-corpus offre également une variété plus importante de "sous-thématiques »- ou de points de vue à l'intérieur de cette thématique : passage du libéralisme au royalisme (ou du moins simulation de ce passage) ; opposition neutre ; ou opposition axiologique :

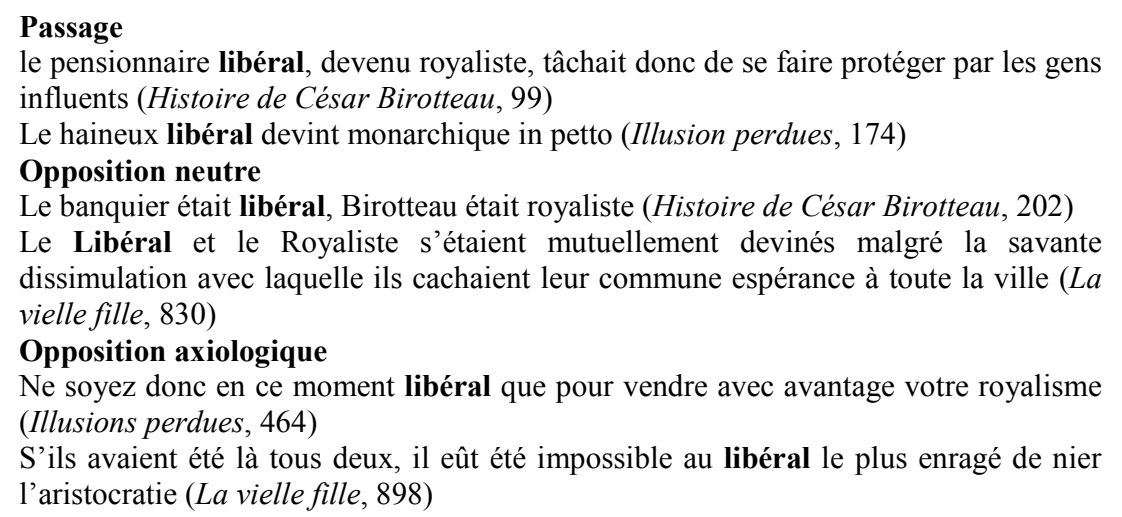

Le passage d'un état (ou situation) à l'autre est attesté par le verbe devenir, marquant parfois une distanciation ironique envers ces revirements. Les éléments de cette thématique peuvent être mis en simple opposition neutre comme c'est le cas dans les exemples appelés ainsi, ou peuvent faire l'objet d'une opposition davantage axiologique, révélant un rapport de dissimulation ou d'euphémisation inhérent au statut de libéral.

Dans le domaine [social], nous trouvons des profilages en relation avec la bourgeoisie :

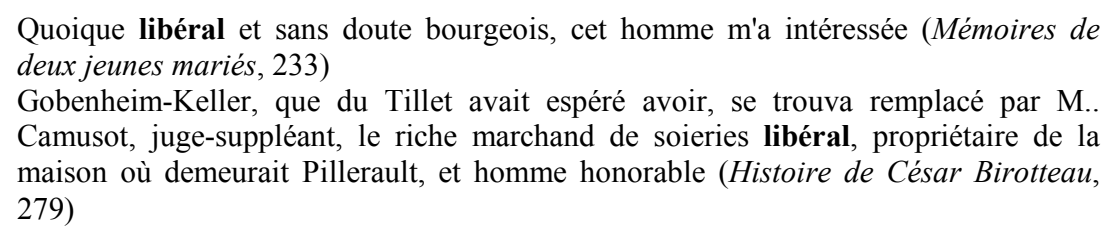

Quant au domaine [moral], nous relevons des profilages selon l'ouverture intellectuelle, la générosité et la libéralité. Par exemple :

\footnotetext{
Restez libéral si vous tenez à votre opinion (La Rabouilleuse, 313)

Mon père se montra d'ailleurs libéral pour tous les frais nécessités par mon éducation, et pour les plaisirs de la vie parisienne (Le médecin de campagne, 541)

Si quelque libéral adroit s'emparait de cette tête vide, il vous causerait des chagrins (Le curé de Tours, 234)

Ottoboni, reprit-il, est un saint homme, il est très secourable, tous les réfugiés

l'aiment, car, Excellence, un libéral peut avoir des vertus! (Gambara, 469)
}

Le motif //ouverture// se stabilise selon les domaines, et le dynamisme permet de rendre compte des combinaisons des sens relatifs aux domaines: libéral se stabilise selon les domaines d'emploi, en intégrant les dimensions connexes et les effets de figements propres aux collocations et à la textualité. Chaque domaine apparaît comme englobant face à celui dont il semble être l'extension : ceci est particulièrement vrai (et déclaré comme tel) pour [politique], qui se veut la synthèse et le prolongement 
des domaines [moral] et [social] ; c'est également vrai pour [social] face à [moral], le positionnement social résultant pour partie dans ces corpus des dispositions morales et intellectuelles (d'ouverture).

Le schéma suivant synthétise les différentes thématiques qui sont mobilisées dans le corpus Stendhal :

/sens

commun/

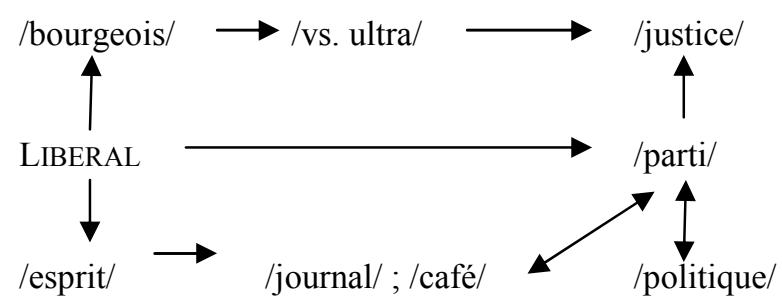

Schéma $n^{\circ} 2: \quad$ Thématiques dans le corpus Stendhal

En comparaison du corpus Balzac, nous remarquons un double mouvement, que nous avions suggéré précédemment: des mécanismes du sens commun opèrent sur ce lexème, et parallèlement une cristallisation des sens vers les acceptions politiques plonge l'objet dans des thématiques qui se lient étroitement. Ainsi nous trouvons à la fois des utilisations de libéral qui ne le créditent pas de sens issus du motif, et également des dynamiques qui anticipent prioritairement sur les thématiques du domaine [politique]. Les constructions sémantiques dans le corpus Stendhal s'intensifient dans le pôle [politique], cristallisant un certain nombre de sens bien particuliers. Les acceptions politisées se déclinent en diverses thématiques, les plus originales par rapport au corpus Balzac étant celles de la /justice/ (souvent spécifiée en «prison ») et du /café/ :

La duchesse rencontra sur l'esplanade de la grosse tour un pauvre libéral prisonnier, qui était venu jouir de la demi-heure de promenade qu'on lui accordait tous les trois jours (La Chartreuse de Parme, 111)

Ces phrases emphatiques et convenues que j'écris avec variations, dans la bonne intention de faire pâlir un préfet qui souffre un café libéral dans sa ville [...] (Lucien Leuwen, T. 3, 364)

D'autres spécificités sont à décrire. Par exemple :

Lui répondait un jeune fabricant libéral, $M$ De Saint-Giraud n'est-il pas de la congrégation? (Le Rouge et le Noir, 150)

Il remarqua l'officier libéral, manchot, qui, placé derrière la vitre verdâtre du cabinet littéraire, tenait un numéro de la tribune et le regarda du coin de l'oeil comme il passait (Lucien Leuwen, T.1, 196) 
Dans ces deux exemples, libéral pourrait être omis sans qu'il y ait une perte de sens : libéral ajoute une précision facultative sur les personnages (dans le second exemple, la récurrence des syntagmes officier libéral manchot dans le texte nous incite à penser cela, même si l'exemple isolé n'est pas forcément très probant). Le positionnement socio-politique des auteurs peut éclairer ces divergences: Stendhal, admirateur et partisan de Napoléon, est favorable au libéralisme de l'époque, ce qui justifie la politisation des emplois. Mais d'un autre côté, cet engagement rend son écriture plus imprégnée des idées libérales, ce qui peut justifier l'insertion plus massive du lexème libéral sans que celui-ci apporte sa dimension informative ou argumentative aux énoncés. Balzac adopte quant à lui une posture qui ressemble davantage à celle d'un observateur, ce qui explique l'intégration des occurrences dans des contextes politiques concrets (parti libéral, bourgeois libéral, libéral opposé à royaliste).

Le motif//ouverture//, qui sous-tend ces différentes thématiques, est donc profilé en insérant l'objet dans différents domaines : [politique], [social] et [moral] :

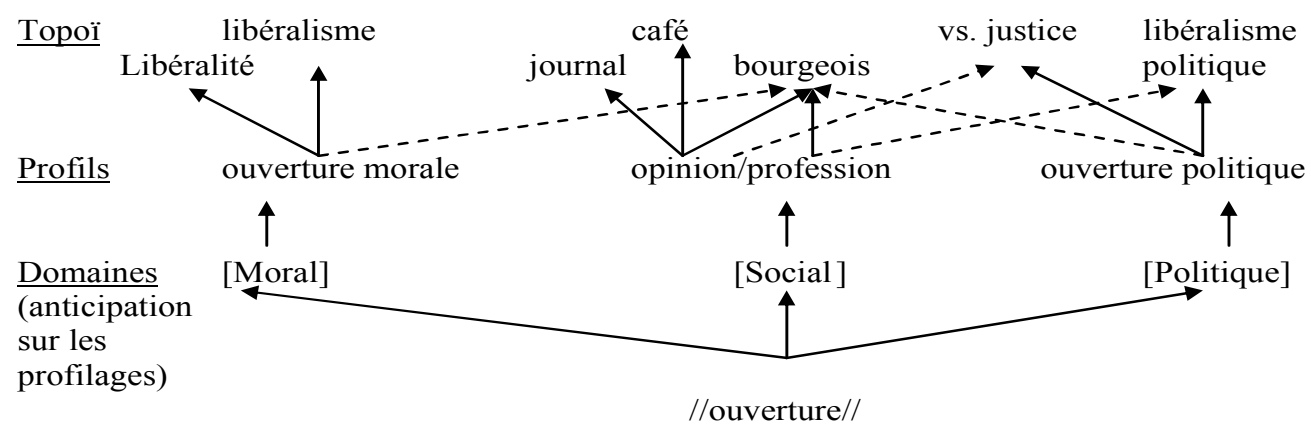

Schéma nº $\quad$ Dynamiques sémantiques de LIBERAL

La stabilisation de formes sémantiques à partir du motif//ouverture// se fait en interaction avec les profils voisins. Ces opérations sont liées aux dynamiques de l'énonciation, qui constituent un niveau intermédiaire entre le motif identifié, et les thématiques. Du coup, cette représentation schématique devient confuse, ce qui est - au-delà de l'aspect purement présentatif des résultats - plutôt rassurant. Nous pouvons certes repérer la motivation de l'objet, les domaines dans lesquels il s'insert, et les profilages, mais l'objet discursif est finalement plongé dans des topiques qui enregistrent les profils en vigueur dans d'autres pôles, rendant en outre possible la richesse sémantique et le potentiel d'emploi de chaque utilisation. Il est intéressant de noter que dans le Grand Dictionnaire Universel de Pierre Larousse (1866), les définitions proposées vont dans le sens de la motivation sémantique que nous identifions, davantage que les sources historiques utilisées précédemment. Tout se passe comme si les discours de lexicographie historique actuels infléchissaient légèrement la perspective diachronique. En effet, libéral(e) est défini en premier lieu dans le $G D U$ par « généreux, qui aime à donner »; en deuxième lieu, il est défini par "Qui est favorable aux libertés » (avec comme exemple Des idées libérales). Dans le même sens, le GDU propose «Qui développe librement les facultés de l'homme » (comme l'éducation libérale). Profession libérale est ensuite définie, par «dans l'exercice de laquelle l'intelligence a plus de part que la main ». En tant que substantif, le GDU définit ce lexème par "personne qui aime à donner », et «partisan de la liberté». Ces définitions, contemporaines aux auteurs étudiés, témoignent de l'argumentativité d'un tel lexème, mais surtout elles rendent compte de la microgenèse sémantique à l'œuvre, et renvoient aux dynamiques de constitution présentes dès le motif. Il est intéressant de noter que le travail réalisé sur ce corpus est finalement en correspondance avec les discours lexicographiques produits à cette époque: ces données s'éclairent mutuellement, et permettent de mieux saisir le sémantisme de libéral(isme). 


\section{Les enjeux argumentatifs des discours contemporains}

Si l'on s'intéresse aux discours des formations politiques contemporaines, le lexème libéral semble faire état d'une profusion de sens. Ceci a été vérifié dans une étude de corpus menée dans les discours prononcés par Chirac, Madelin et Le Pen de 1997 à 2004 ${ }^{5}$. Dans tous ces discours, nous avons des constructions sémantiques très différentes, qui s'expliquent par les différences de positionnement énonciatif. Cependant, toutes sont sous-tendues par le motif//ouverture//.

\subsection{Dans le discours de Le Pen : variation selon la prégnance de /louverture//}

Chez cet énonciateur, il apparaît que libéral retient une dimension collective de la liberté, et en rejette la dimension individuelle et immorale : le topos « le libéralisme apporte la liberté » est acceptable dans une certaine mesure, alors que le topos «le libéralisme rend libre » est réfuté. Le caractère scalaire permet ainsi de poser l'apport morphémique dans la construction globale de la forme sémantique : le morphème est «activé » mais son intensité doit être très faible pour que le sens soit acceptable par la communauté discursive représentée par le candidat. C'est pourquoi Le Pen utilise beaucoup le préfixe ultra qui atteste presque morphologiquement de la trop forte intensité de l'objet, ce qui est en fait la majorité des cas. Par exemple :

le Code de la nationalité et le droit d'asile vont être réformés dans un sens ultralibéral (26 sept. 1997)

La Sécurité Sociale, qui sera demain entre les mains de Bruxelles, risque de passer à

la moulinette de l'idéologie ultra-libérale de la Commission (17 janv. 1999)

Le schéma suivant modélise les constructions sémantiques, selon l'intensité et le positionnement de la motivation (on notera que dans les contextes défavorables à l'idéologie libérale, libéralisme est préféré à libéral: sans entrer ici dans le détail, le processus apparaît comme néfaste, alors que la qualification d'objets de libéral(e) est moins négative) :

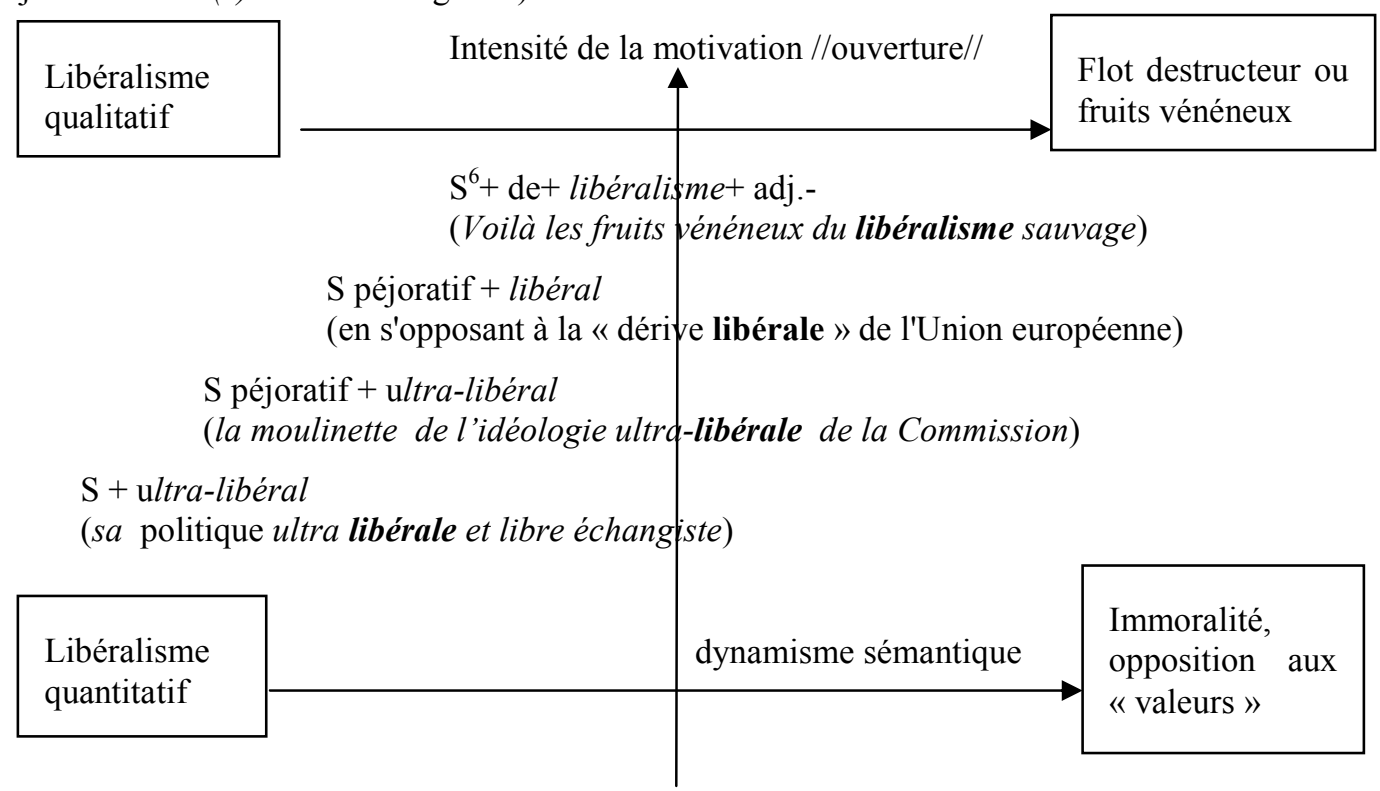

Schéma ${ }^{\circ}$ 4: $\quad$ Dynamiques sémantiques dans le corpus Le Pen

C'est donc bien l'intensité de l'activation du motif //ouverture// qui préside à toutes ces dynamiques, que son intensité soit quantitative (trop d'ouverture) ou qualitative (une ouverture trop néfaste). 


\subsection{Dans les discours de Chirac : //ouverture// selon le contexte d'énonciation}

Chirac synthétise le point de vue comparativement à ce que recouvre //ouverture// dans le contexte énonciatif dans lequel il produit son discours. Ainsi lorsque //ouverture// s'actualise sous forme d'un principe, de manière absolue (la Liberté), la composante morphémique permet une dynamique de constitution qui va créer un objet très positif. En effet, dans des contextes qui connaissent un déficit d'ouverture, Chirac construit des objets qui ne peuvent pas renvoyer aux côtés négatifs, même s'ils existent et sont exprimés dans d'autres contextes. Il y aurait en effet une sorte d'indécence à critiquer l'excès d'ouverture alors même que les interlocuteurs en sont privés. Par contre si //ouverture// s'active en points de vues plus particuliers, on peut rencontrer des dynamiques qui déconstruiront cet aspect favorable qui créditait l'objet. En effet, si l'on quitte le principe et l'absolu, l'ouverture peut être excessive et néfaste, comme Le Pen ne cesse de le rappeler. Ainsi ce sera surtout quand Chirac est face à des entités qui ont une conception plus intensive de la liberté qu'il se défendra d'être trop libéral, ou favorable au libéralisme. Sans y être opposé, il parlera de régulation ou de compensation. Le schéma suivant recense les divers contextes d'emplois :

Pays qui accèdent à la démocratie/Pays en Voie de Développement

(parce qu'on ne peut pas être un système libéral sur le plan économique et puis rester fermé sur le plan politique)

Dans les discours sur le politique

(Le professionnel libéral se distingue par le respect de règles fondamentales parfois héritées d'un long passé)

Dans les discours sur les politiques économiques

(Ils sont un pays libéral, ce n'est pas l'Etat qui décide d'un embargo économique)

Discours qui intègrent l'Europe/ le Monde

(Je l'observe et je l'écoute dans toutes les discussions internationales, c'est un homme très très libéral)

Evaluation

Schéma ${ }^{\circ} 5: \quad$ Les dynamiques du sens chez Chirac

\subsection{Dans les discours de Madelin: //ouverture// à tous points de vue}

On trouve dans ce sous-corpus une proportion plus importante de libéral(e), employé comme qualificatif, que de libéralisme: il est le qualificatif de politique (6 fois), de pensée (4 fois), de voie ( 2 fois), de professions ( 2 fois), etc., alors que le corpus fournit seulement 5 attestations de libéralisme. C'est donc qu'une intentionnalité sous-tendrait sa conception de /liberté/, ce qui s'intègre comme motivation et se manifeste en syntagme. Chez cet énonciateur libéral, //ouverture// est profilé selon trois domaines, qui rejoignent les grands thèmes libéraux. Des constructions liées à la /responsabilité/autonomie/ plongent l'objet dans les thématiques liées à l'individualisme : l'homme est responsable de ses actes et de son destin, et le libéralisme lui donne les moyens de son épanouissement. L'homme, vu comme la valeur absolue, semble crédité d'une éthique de la responsabilité, qui renforce cette idée individualiste :

Une politique libérale c'est une politique qui part d'un principe : la confiance dans l'individu libre et responsable. [...] Une politique libérale est une politique de citoyens responsables (5 av. 1997)

//Ouverture// comme /mouvement/modernité/ ouvre sur les thématiques de l'avenir et du dynamisme, puisque l'objet entre en adéquation avec les aspiration des individus et $\mathrm{du}$ monde. En outre, ces 
thématiques, qui sont sociologiquement marginales (au regard des scores électoraux), sont justement peu répandues parleur caractère précurseur et non-conformiste :

Il faut avoir l'audace d'un changement fort, d'une alternance moderne, d'une alternance libérale (21 sept. 1999)

Enfin, //ouverture// orienté vers la /justice/ conduit à la référence à la fameuse main invisible d'Adam Smith : le libéralisme conduit à une situation de justice parfaite, dans laquelle chacun trouve son intérêt, et dans laquelle l'intérêt commun vient justement de l'équilibre métaphoriquement établi par cette main invisible libérale :

Le libéralisme est le produit d'une quête millénaire du vrai et du juste fondée sur une conception de l'universalité humaine (29 juin 2000)

Dans ces discours, libéral est crédité d'une valeur très positive, que le motif//ouverture// explique.

Pour schématiser les différences relevées chez ces trois énonciateurs, nous pouvons représenter les aboutissements de dynamiques selon les deux axes suivants (évaluation de l'objet et positionnement quantitatif ou qualitatif) :

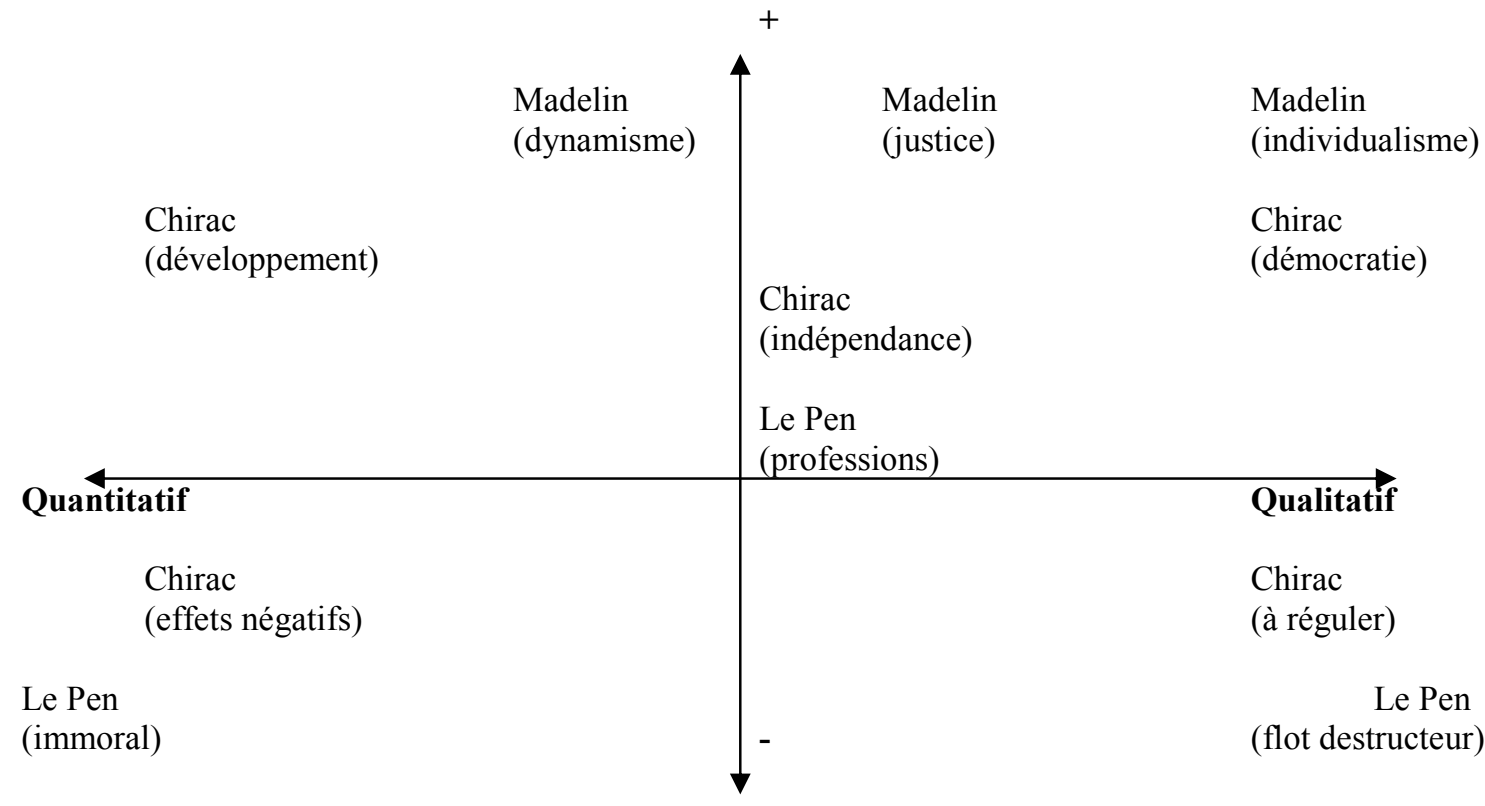

Schéma n ${ }^{\circ}$ : $\quad$ Aboutissement des différentes dynamiques

Nous obtenons ainsi une topographie des lieux relatifs à libéral(isme) dans le discours dit «de droite » (au moins une partie représentative, qu'elle soit traditionnelle, libérale ou extrême) aux alentours des années 2000. Il pourrait sembler problématique de se trouver face à une telle diversité des sens. Mais nous l'avons vu, en caractérisant les parcours de sens plutôt qu'en focalisant l'analyse sur les aboutissements, cette représentation peut devenir éclairante.

Avec l'aide de la description historique et étymologique, il semble judicieux de considérer que le motif est //libre/sans entrave//: mais pour être plus précis, et remonter encore en amont, le motif //ouverture// fourni par le corpus Frantext paraît bien correspondre, et permet de rendre compte des anticipations qui peuvent être faites par les candidats : 
A. MADELIN

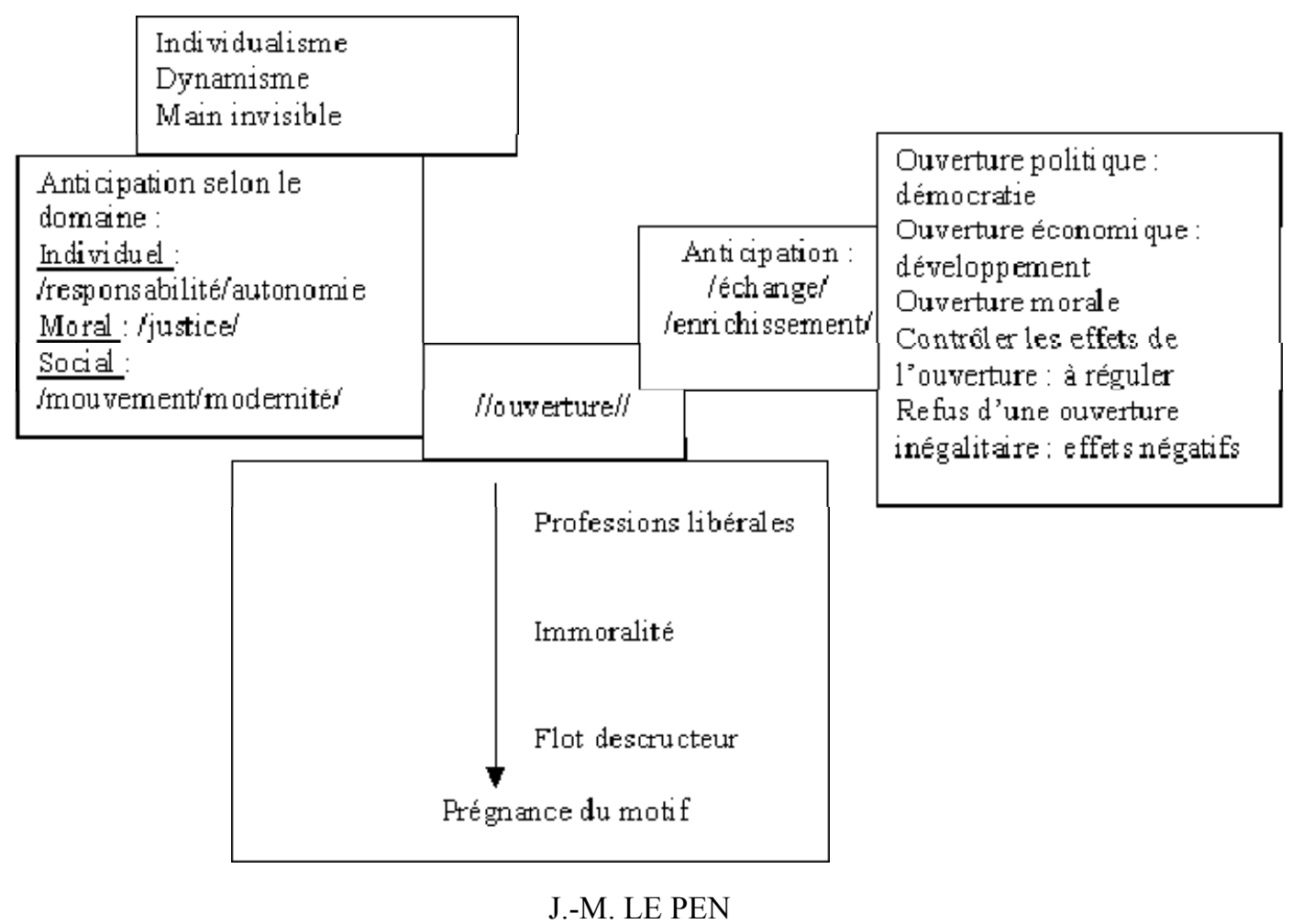

Schéma ${ }^{\circ} 7$ : Motif de libéral, anticipation des profilages, et topiques discursives

Comme pour le schéma synthétique concernant le corpus précédent, le dynamisme sémantique s'appuie sur le motif //ouverture// que nous avons identifié : pour chaque candidat, l'individuation de ce motif établit une anticipation sur les opérations de profilage: selon certaines dimensions liées au positionnement énonciatif pour A. Madelin et J. Chirac, et selon l'intensité de la motivation chez J.-M. Le Pen. En outre, en amont, ce schéma confirme l'anticipation générale qui opère sur libéral(isme), en le positionnement le plus souvent dans le domaine socio-économique.

\section{Conclusion}

Si les usages de libéral(isme) conduisent actuellement à des discours centrés sur les domaines économiques et politiques, la prise en compte de l'évolution des sens, des champs d'application, et de l'axiologisation, permettent de mieux saisir la particularité des emplois de ce mot. Le motif //ouverture// que nous avons identifié, en relation avec la morphologie et l'étymologie, permet de comprendre le positionnement des dynamiques de constitution du sens en discours, à la fois dans les discours contemporains, mais également à divers paliers, comme celui du $19^{\text {ème }}$ siècle utilisé ici. Cette analyse permet de saisir ce qui est en jeu dans l'utilisation de libéral(isme), sans pour autant privilégier les facteurs contextuels et la fréquence d'emplois. Passé d'un sens proche de généreux et ouvert d'esprit, libéral fait à présent l'objet d'enchainements souvent péjoratifs : nous ne pouvons prétendre savoir ce qu'il en sera dans l'avenir. Il ne faut cependant pas oublier que l'usage politique du terme libéral reste actuellement lié à la théorie économique néo-libérale préconisée par Friedman, Becker ou Hayek, et mise en oeuvre par Reagan et Thatcher. Ainsi un travail sur l'évolution des rapports entre l'économie et la politique au cours du $20^{\text {ème }}$ siècle pourrait éclairer notre problématique à propos des usages contemporains. 


\section{Références bibliographiques}

Anscombre, J.-C. (dir.). (1995). Théorie des topoï. Paris : Kimé.

Cadiot P. (1997). Sur l'indexicalité des noms, 243-269, dans D. Dubois (ed.), Catégorisation et cognition, Paris : Kimé.

Cadiot P. et Visetti Y.-M. (2001-2). Motifs, profils, thèmes : une approche globale de la polysémie, Cahiers de lexicologie, 79, 5-46.

Foucault M. (1969). L'archéologie du savoir. Paris : Gallimard.

Lebas F. (1999). L'indexicalité du sens et l'opposition "en intension »/ " en extension ». Thèse de doctorat, Université Paris 8.

Lebas F. et Cadiot P. (éds). (2003). La constitution extrinsèque du référent, Langages, 150.

Longhi J. (2006). L'étymologie en sémantique : motifs et motivations des objets du discours, Bulag, 31, 89-100.

Longhi J. (2007). L'objet discursif intermittent : construction d'une forme sémantique et évolution des topoï dans un corpus de Presse, 149-163, dans G. Cislaru, O. Guerin, K. Morim, E. Née, T. Pagnier, M. Veniard, M. (éds), L'acte de nommer - Une dynamique entre langue et discours, Paris : Presses Sorbonne Nouvelle.

Longhi J. (2008). Objets discursifs et doxa : essai de sémantique discursive. Paris : l'Harmattan.

Longhi J. (à paraître). Quel petit livre argumentatif au fond de la cour ? Eléments de description sémantique argumentative de livre. Langages (P. Larrivée dir.).

Merleau-Ponty M. (1969). La prose du monde. Paris : Gallimard

Sarfati G.-E. (1996). La sémantique : de l'énonciation au sens commun. Éléments d'une pragmatique topique. Texto ! décembre 2004 [en ligne] Disponible sur : http://www.revue-texto.net/Inedits/Sarfati/Sarfati_Semantique.html

Dictionnaire de la conversation et de la lecture. (1867). Paris : Librairie de Firmin Didot frères, fils et $C^{\text {ie }}$.

Dictionnaire Historique de la langue française (tome I). (2001). Paris : Le Robert.

Grand Dictionnaire Universel du XIXème siècle. (1866), par Pierre Larousse.

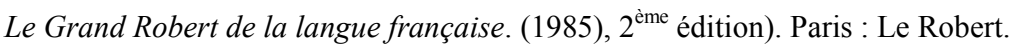

Le Robert, dictionnaire d'aujourd'hui. (1992). Alain Rey (dir.). Paris : France Loisir.

Le Trésor de la Langue Française Informatisé (TLFI), accessible depuis http://atilf.atilf.fr/dendien/scripts/tlfiv4/showps.exe?p=combi.htm;java=no

\footnotetext{
${ }^{1}$ Voir par exemple Lebas et Cadiot $(2003,5):$ «Le monde est une constitution compatible avec l'expérience parce qu'il est constitué par l'expérience. Plus particulièrement, la constitution des référents leur est extrinsèque, elle est fondée sur notre expérience. La solution générale de l'articulation sens-référence est alors énonçable avec une extraordinaire simplicité : les objets de la parole sont propres à l'activité linguistique en tant qu'ils sont en partie constitués par la dynamique langagière, mais sont aussi les mêmes que ceux auxquels le langage réfère ».

${ }^{2}$ Nous nous concentrons dans un premier temps sur libéral, puisque libéralisme est utilisé de manière très marginale jusqu'à la fin du $19^{\text {ème }}$ siècle : l'attention portée au domaine économique au cours du $20^{\text {ème }}$ siècle explique la concurrence entre libéral et libéralisme dans les discours du contemporains, comme ceux analysés dans la $3^{\text {ème }}$ partie.

${ }^{3}$ Ces deux auteurs étant considérés comme manifestant des positionnements socio-politiques différents, nous cherchons à montrer la manière dont cette différence se manifeste linguistiquement. Ce choix nous permet également d'avoir un aperçu des usages en cours au 19ème siècle.

${ }^{4}$ Les numéros de pages sont ceux fournis par Frantext.

${ }^{5}$ D'un point de vue pratique, la constitution d'un corpus pour ces candidats est plus aisée que pour d'autres (accès aux discours sur les sites des partis ou de l'Elysée notamment); d'un point de vue scientifique, il nous paraissait
} 
davantage pertinent de saisir les enjeux propres à l'utilisation d'un même objet pour des candidats situés dans le même « hémisphère » politique, afin de mettre en valeur des différences argumentatives et sémantiques précises.

${ }^{6} \mathrm{~S}=$ substantif ; adj- = adjectif péjoratif 